\title{
Maintenance of youth-like processing protects against false memory in later adulthood
}

\author{
Yana Fandakova ${ }^{1}$, Ulman Lindenberger, Yee Lee Shing* \\ Center for Lifespan Psychology, Max Planck Institute for Human Development, Berlin, Germany
}

\section{A R T I C L E I N F O}

\section{Article history:}

Received 19 November 2013

Received in revised form 15 September 2014

Accepted 14 October 2014

Available online 22 October 2014

\section{Keywords:}

Episodic memory

Aging

False memory

Cognitive control

fMRI

\begin{abstract}
A B S T R A C T
Normal cognitive aging compromises the ability to form and retrieve associations among features of a memory episode. One indicator of this age-related deficit is older adults' difficulty in detecting and correctly rejecting new associations of familiar items. Comparing 28 younger and 30 older adults on a continuous recognition task with word pairs, we found that older adults whose activation patterns deviate less from the average pattern of younger adults while detecting repaired associations show the following: (1) higher overall memory and fewer false recognitions; (2) stronger functional connectivity of prefrontal regions with middle temporal and parahippocampal gyrus; and (3) higher recall and strategic categorical clustering in an independently assessed free recall task. Deviations from the average youngadult network reflected underactivation of frontoparietal regions instead of overactivation of regions not activated by younger adults. We conclude that maintenance of youth-like task-relevant activation patterns is critical for preserving memory functions in later adulthood.
\end{abstract}

(C) 2015 Elsevier Inc. All rights reserved.

\section{Introduction}

Normal cognitive aging is associated with a decline in the ability to form and retrieve associations among different features of an episode (Old and Naveh-Benimain, 2008; Shing et al., 2010). This general trend of declining associative memory is accompanied by massive individual differences in rates of decline (Ghisletta et al., 2012; Lindenberger and Ghisletta, 2009; Persson et al., 2012). As a result, associative memory is preserved in some older individuals, but not in others (Fandakova et al., 2012). Little is known about the mechanisms driving this heterogeneity (Barulli and Stern, 2013; Nyberg et al., 2012). The general goal of this study is to examine the degree to which individual differences in neural activation and connectivity are related to individual differences in associative memory and false remembering in later adulthood.

Evidence is accumulating that age-related memory deficits are especially pronounced when individuals are required to remember specific contextual details instead of isolated items (NavehBenjamin, 2000; Spencer and Raz, 1995). For example, when

\footnotetext{
* Corresponding author at: Center for Lifespan Psychology, Max Planck Institute
for Human Development, Lentzeallee 94,14195 Berlin, Germany. Tel.: +49 30824

* Corresponding author at: Center for Lifespan Psychology, Max Planck Institute
for Human Development, Lentzeallee 94, 14195 Berlin, Germany. Tel.: +49 30824 06 436; fax: +49308249939.

E-mail address: yshing@mpib-berlin.mpg.de (Y.L. Shing).

1 Present address: Center for Mind and Brain, University of California, Davis, CA, USA and Helen Wills Neuroscience Institute, University of California, Berkeley, CA, USA.
}

0197-4580/\$ - see front matter (c) 2015 Elsevier Inc. All rights reserved.

http://dx.doi.org/10.1016/j.neurobiolaging.2014.10.022 investigating memory for word pairs in 278 adults (18-85 years), Bender et al. (2010) found that older participants were not only less likely to correctly endorse studied word pairs but were also more likely to falsely endorse repaired associations in which the original words were studied in different configurations (see also Shing et al., 2008). In fact, a direct comparison of the two effects revealed a stronger association between age and false endorsement of repaired associations than between age and failure to recognize intact associations. At the same time, this study revealed a considerable amount of variability among individuals of the same age. For example, in the group of 60- to 80-year-olds, some individuals were almost perfect in correctly detecting repaired associations whereas others wrongly endorsed repaired associations in more than $70 \%$ of the cases (see Fig. 1, Bender et al., 2010). This observation is in line with longitudinal observations, indicating increasing heterogeneity of cognitive functions with advancing adult age (de Frias et al., 2007; Ghisletta et al., 2012; Lindenberger and Ghisletta, 2009). Given the notable difficulties of older adults to detect repaired associations of familiar features, the specific goal of this study was to characterize the neural mechanisms underlying heterogeneity in older adults' tendency to falsely recognize associations they have not encountered before. This topic is of great importance for everyday life because it may result in greater susceptibility to misinformation (Jacoby and Rhodes, 2006).

Compared with the detection of novel or intact associations, repaired associations are expected to engage mnemonic control to a greater degree, as single items are highly familiar and need to be 


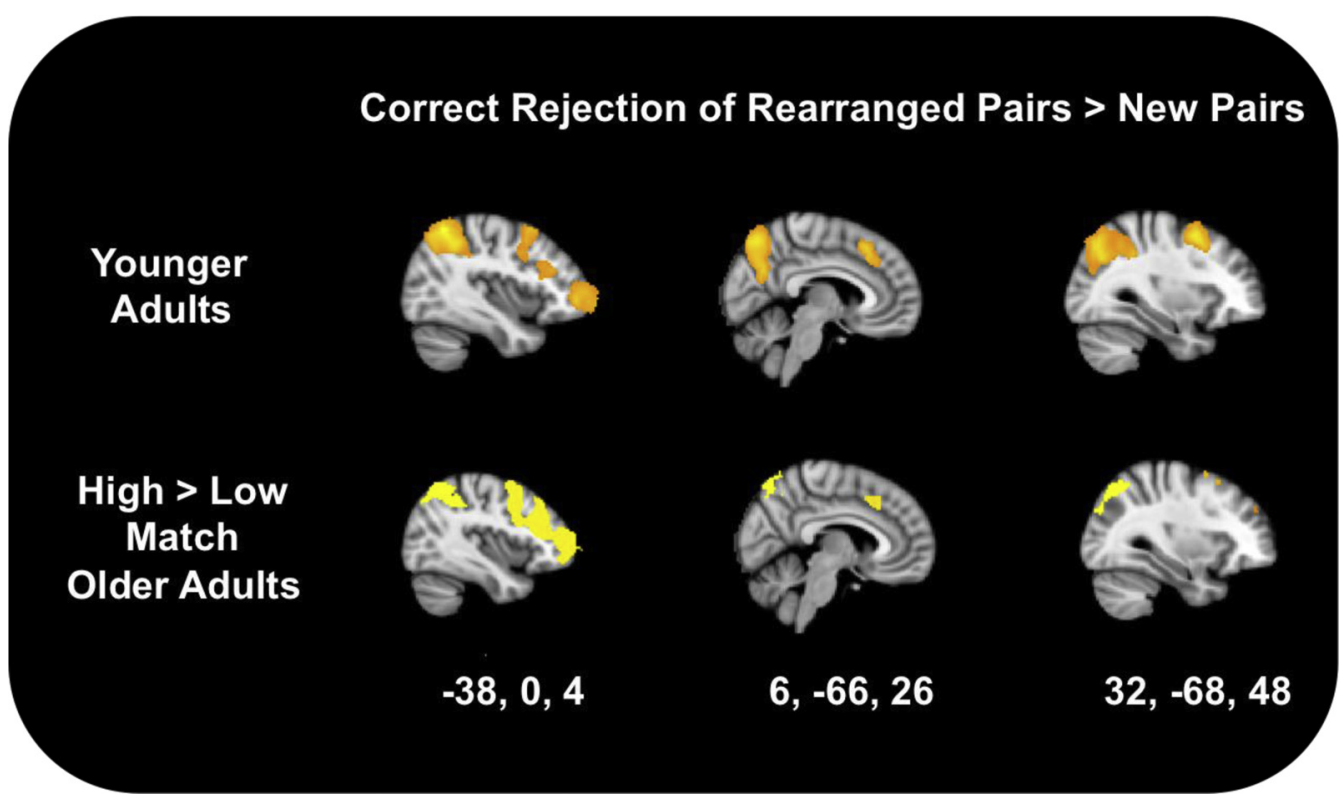

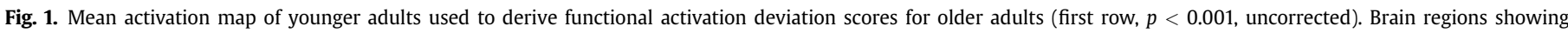

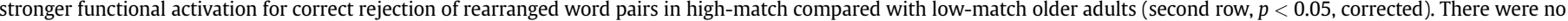
regions in which low-match older adults showed stronger activation $(p<0.05$, corrected). Coordinates $(\mathrm{x}, \mathrm{y}, \mathrm{z})$ in MNI standard space.

closely monitored to avoid memory errors (Mitchell and Johnson, 2009). Accordingly, correct rejection of repaired associations typically engages lateral prefrontal cortex (PFC; Lepage et al., 2003), which is known to support mnemonic control over memory contents (Simons and Spiers, 2003).

PFC undergoes declines in both gray and white matter volume in later adulthood (Raz et al., 2005). Compared with younger adults, older adults have shown lower PFC activation during encoding or retrieval of past episodes (Wang et al., 2009). However, other studies have demonstrated additional PFC activation in older adults for memory tasks (Cabeza and Dennis, 2013). In addition, a number of studies have suggested that later adulthood is associated with different patterns of task-related functional connectivity of PFC regions (Daselaar et al., 2006) that are related to individual differences in cognitive performance (Nagel et al., 2011). There is some debate about whether these functional differences reflect corollaries of brain aging (Nyberg et al., 2012), compensatory reactions to age-related declines in posterior brain regions (Cabeza and Dennis, 2013), or the engagement of more neural resources at lower difficulty levels in older adults (Reuter-Lorenz and Cappell, 2008). In any case, the claim that between-person differences in PFC volume, functional activation, and connectivity among older individuals increase from early to later adulthood appears to be well founded (Lindenberger et al., 2013). Thus, the increased heterogeneity in functional activation and connectivity of these regions may contribute to individual differences in associative memory in later adulthood.

The few neuroimaging studies that have examined age differences in encoding or retrieval of associative information provide initial support for PFC contributions to age differences in associative memory (Dennis et al., 2008a; Fandakova et al., 2014; Ford et al., 2010; Giovanello and Schacter, 2012). However, individual differences in neural activation or behavioral performance have not been addressed in these studies. Here, we go beyond mean age differences in neural mechanisms of associative recognition by directly relating differences in neural activation to the observed heterogeneity in associative memory among older adults. We focus our analyses on the correct rejection of repaired associations, a process that requires increased mnemonic control (Mitchell and Johnson, 2009) and poses a substantial challenge to older adults (Jacoby and Rhodes, 2006). Thus, the goals of the present study were to examine the following: (1) the extent to which individual differences in functional activation among older adults, defined as a relative match to functional activations in younger adults during the detection of repaired associations, are related to differences in memory performance; (2) differences between high- and lowmatch older adults in task-related functional connectivity that may reflect interindividual differences in strategic control of mnemonic content; and (3) whether between-person differences observed on the target memory task generalize to other tasks that presumably require similar mnemonic processes.

To address these issues we scanned younger and older adults working on a continuous recognition task. The task consisted of 3 runs. Within each run, the same set of word pairs was presented once or twice, along with novel and repaired word pairs (for details, see Fandakova et al., 2014). Participants' task was to detect word pair repetitions within the current run while rejecting pairs that were either appearing for the first time within the run, novel, or repaired. Repaired associations were composed by rearranging pairs that had been presented in every run. We hypothesized that correctly rejecting such associations would become easier across runs. This manipulation enabled us to investigate how changing task demands modulated processes involved in associative recognition. Using this paradigm, we previously reported robust mean age differences in a frontal-parietal network involved in the correct rejection of repaired associations (Fandakova et al., 2014). The goal of the present analysis was to delineate the mechanisms underlying individual differences among older adults. Following a method introduced by Düzel et al. (2011), we characterized each older individual's activation pattern in terms of the degree to which it matched the average functional activation pattern of younger adults. Based on recent findings that older adults who showed less functional deviation from younger adults in encoding-related brain activity also showed higher recollection (Düzel et al., 2011), we expected that preserved ability to detect repaired associations of familiar words would be associated with the preservation of 
youth-like activation patterns. Furthermore, we examined how individual differences in the preservation of youth-like task-related activations relate to individual differences in functional connectivity. Finally, based on our initial observation that the continuous recognition task engages a frontoparietal network associated with mnemonic control (Fandakova et al., 2014; Simons and Spiers, 2003), we expected that an independently assessed free recall task with relatively high demands on mnemonic control (Becker and Lim, 2003) might be associated with individual differences in functional activation.

\section{Methods}

\subsection{Participants}

Twenty-eight younger (mean $[\mathrm{M}]_{\text {age }}=24.92$ years, standard deviation $[\mathrm{SD}]_{\text {age }}=1.84,15$ females $)$ and 30 older $\left(\mathrm{M}_{\mathrm{age}}=\right.$ 72.28 years, $\mathrm{SD}_{\text {age }}=2.01,13$ females) adults participated in the experiment. All participants were healthy right-handed adults without history of neurologic or psychiatric disorders.

\subsection{Materials}

Stimuli were 414 German nouns used to form 207 unrelated word pairs. A set of 132 word pairs was randomly selected to serve as stimuli for younger adults. From these, a subset of 102 word pairs was randomly assigned to older adults. Of the remaining word pairs, 75 were used as novel pairs for both age groups. The stimulus number differed between the age groups to render the task manageable for both age groups.

\subsection{Procedure}

First, participants were prefamiliarized to 132 word pairs (younger adults) or 102 word pairs (older adults) by asking them to provide living or nonliving judgments on each pair. Following the familiarization phase, participants were scanned during 3 consecutive runs of the continuous recognition task. In each run, the complete set of prefamiliarized word pairs was presented once along with 25-novel word pairs that had not been seen before in the experiment. In addition, one-third of the prefamiliarized word pairs (44 for younger adults and 34 for older adults) were repeated within the ongoing run. A different third was repeated in each run, such that by the end of the task each prefamiliarized word pair was repeated exactly once across all 3 runs. Another one-third of the word pairs reappeared in the ongoing run as a repaired association, or rearranged pairs ( 44 for younger adults and 34 for older adults), such that the left word of a pair that has previously been presented for the first time in the ongoing run was presented with the right word from another pair previously presented for the first time in the ongoing run. These rearranged pairs were unique and were never repeated across runs. The word pairs used to create the repaired associations did not overlap with those used as repeated pairs within the same run. Prefamiliarized, repeated, rearranged, and novel word pairs were presented for 3 seconds in a randomized order, followed by a jittered fixation period (500-3000 ms, optimized using Optseq 2; Dale, 1999). Participants were instructed to indicate, for each word pair, if they saw the particular word pair for the first or second time in the ongoing run. They were instructed to press "sure new" or "unsure new" if the particular word combination was seen for the first time in the ongoing run, and "unsure old" or "sure old" if it was repeated in the ongoing run. Before the task, the participants were informed about the different word pair types and practiced the task with different word pairs outside the scanner.

\subsection{Functional magnetic resonance imaging methods}

\subsubsection{Data acquisition}

Scanning was conducted using a 3T Siemens Trio Magnetom. Thirty-six functional slices were acquired using an echo planar imaging sequence (repetition time, $2000 \mathrm{~ms}$; echo time, $30 \mathrm{~ms}$; field of view, $216 \mathrm{~mm} ; 72^{2}$ matrix; and $3 \mathrm{~mm}^{3}$ voxel size). Structural data consisted of a high-resolution $\mathrm{T}_{1}$-weighted sequence (repetition time, $1550 \mathrm{~ms}$; echo time, $2.34 \mathrm{~ms}$; inversion time, $900 \mathrm{~ms}$; $350 \times 263 \times 350$ matrix; $1 \mathrm{~mm}^{3}$ voxel size).

\subsubsection{Functional magnetic resonance imaging analysis}

Data were preprocessed and analyzed using FEAT in FMRIB software library. Preprocessing included the following: nonbrain tissue removal, slice time and motion correction, time-series prewhitening, spatially smoothed using a 8-mm Gaussian FWHM, and normalization to the MNI space using FLIRT. Low-frequency artifacts were removed by a high-pass temporal filter (sigma $=50$ seconds).

Individual time series were modeled with regressors of interest including correct responses to rearranged and novel pairs. Regressors of no interest were specified to model first presentations of prefamiliarized word pairs within a run, repeated pairs, and error trials. To identify regions involved in the detection of repaired associations, a contrast of correct rejections (CR) to rearranged pairs and novel pairs was performed per run per subject (i.e., CR rearranged $>C R$ novel). In this contrast, participants gave the same overt response (i.e., responding "new"), but it was expected that repaired associations would involve more strategic monitoring of memory contents, thus allowing us to isolate the mnemonic control network. Temporal derivatives were added in all analyses. Contrast images, after resampling to $2 \mathrm{~mm}$ isotropic voxels in MNI space, were submitted to a within-subject fixed-effects analysis across runs. Higher-level analysis was carried out using a mixed-effects model in FMRIB software library FLAME.

\subsubsection{Functional activation deviation score}

A functional activation deviation score was derived for each individual to quantify how much his or her functional brain activation matched the younger adult group during the detection of repaired associations following the procedure described in Düzel et al., (2011). First, we assessed the average younger adults' functional activation network involved in the detection of repaired associations by comparing brain activation for $\mathrm{CR}$ rearranged $>\mathrm{CR}$ novel. All activated clusters ( $p<0.001$, uncorrected) were included to form the average younger adults' activation mask. Second, for each older adults we used the activation map of the contrast CR rearranged $>$ CR novel to compute the individual mean $T$-value of all voxels that fall within the average younger adults' activation mask ( $T_{\text {inside mask }}$ ). Similarly, we computed the mean $T$-value of all voxels outside of the average younger adults' activation mask ( $T_{\text {outside }}$ mask $)$. Finally, a functional activation deviation score was derived for each older adult by computing $T_{\text {outside mask }}-T_{\text {inside mask }}$ (cf. Düzel et al., 2011). Thus, a large negative score indicates high match to younger adults in terms of functional activity during correct detection of repaired associations.

To investigate differences in functional connectivity, we conducted a psychophysiological interaction analysis (PPI; Friston et al., 1997). A cluster in left anterior PFC was selected as a seed region based on our previous findings that this region plays a central role in mnemonic control of familiar information (Fandakova et al., 2014; see also Badre and Wagner, 2005). The mask used in the PPI analysis was identical to the left anterior PFC region of interest (BA10) described in Fandakova et al. (2014). It was defined as all active voxels across younger and older adults that lay within the specific anatomic region of the Harvard-Oxford cortical atlas. 
Because our previous analyses demonstrated that this brain area is particularly sensitive to memory monitoring in the modified continuous recognition task, using it as a seed region for the present PPI analyses allowed us to examine individual differences in functional connectivity related to mnemonic control. Three regressors were used in the PPI analyses-time course in left anterior PFC, task contrast of $C R$ rearranged $>C R$ novel, and a vector product of the first 2 regressors representing the psychophysiological interaction. Thus, we identified voxels across the brain that have a stronger relationship with the time course in anterior PFC during correct rejection of rearranged as opposed to novel pairs. Given that psychological (i.e., task contrast) and physiological (i.e., seed region time course) components are explicitly modeled, the psychophysiological interaction explains variance beyond the variance explained by the main effects of task and physiological correlation.

Monte Carlo simulations (http://www2.bc.edu/ slotnics/ scripts.htm) were used to define the cluster extend threshold needed to control for multiple comparisons. An individual voxel threshold of $p<0.01$ was used in combination with a cluster threshold of 19 voxels $\left(2 \times 2 \times 2,152 \mathrm{~mm}^{3}\right)$ to correct for multiple comparisons at $p<0.05$ across the whole brain. Additional parameters used in the simulations included brain volume of a $72 \times$ $72 \times 36$ matrix and estimated spatial autocorrelation of $14.91 \mathrm{~mm}$ FWHM (effective smoothness estimated from contrast map), for a total of 10,000 simulations. Group difference maps were inclusively masked with the map of each group to confirm that the resulting differences were related to the expected activity differences between rearranged and novel pairs in each of the examined groups. All reported post hoc comparisons were corrected for multiple comparisons using Bonferroni correction.

\section{Results}

3.1. Functional activation for detection of repaired associations: characterizing older adults based on the average younger adults' network

In younger adults, successful rejection of repaired associations engaged left anterior and inferior frontal gyrus, bilateral superior frontal gyrus, superior parietal lobe, and the paracingulate gyrus (Fig. 1; Table 2). This average activation map including a frontoparietal network was used to compute functional activation deviation scores for older adults (see Section 2.4.3).

Older adults' functional activation deviation scores ranged from -1.95 to $0.33(\mathrm{M}=-0.7$, standard error [SE] $=0.11)$ and represented the degree to which each individual's task-related activation pattern matched the younger adults' network (i.e., more negative scores mean higher match). To further characterize the differences in the present sample, we formed groups of low- and high-match older adults based on a median split of the computed deviation scores. The high-match group $(\mathrm{N}=15)$ was characterized by stronger agreement or match to younger adults, with scores ranging between -1.95 and $-0.69(\mathrm{M}=-1.18, \mathrm{SE}=0.11)$. The lowmatch group $(\mathrm{N}=15)$ was characterized by less agreement or lower match to the younger adults' network, with scores ranging between -0.66 and $0.33(\mathrm{M}=-0.23, \mathrm{SE}=0.08)$. High- and lowmatch groups did not differ in mean age or gender distribution, but high-match older adults had superior performance on vocabulary knowledge and executive functioning tests (see Table 1).

\subsection{Memory differences between high-match and low-match older adults}

A repeated-measure analysis of variance (ANOVA) on overall recognition scores (hits for repeated pairs minus false alarms for rearranged pairs) with task run as a within-subject factor and match group (low vs. high) as a between-subject factor revealed a significant main effect of run, $F(2,56)=10.46, p=0.001, \eta_{p} 2=0.27$, reflecting the increase of associative recognition performance across runs (Fig. 2A, younger adults plotted only for reference). The main effect of match group was reliable, $F(1,28)=7.72, p=0.01, \eta_{p} 2=0.22$ such that overall recognition was higher in the high-match older adults $(\mathrm{M}=0.27)$ compared with the low-match older adults $(\mathrm{M}=0.15)$. Most importantly, the match group $\times$ task run interaction was also significant, $F(2,56)=7.77, p=0.001, \eta_{p} 2=0.22$, indicating that associative recognition changed differentially in the 2 match groups. Follow-up comparisons revealed that although high- and low-match older adults did not differ in the first run of the task, $t(28)=-0.05, p=0.96$, $d=0.02\left(\mathrm{M}_{\text {high match }}=0.15, \mathrm{M}_{\text {low match }}=0.15\right)$, high-match older adults outperformed the low-match older adults in the second, $t(28)=2.61$, $p=0.02, d=0.95\left(\mathrm{M}_{\text {high match }}=0.28, \mathrm{M}_{\text {low match }}=0.14\right)$, and third run, $t(23.5)=3.46, p=0.002, d=1.27\left(M_{\text {high match }}=0.38, \mathrm{M}_{\text {low match }}=0.17\right)$.

To assess whether the observed match group differences in overall recognition may be related to differences in the propensity to falsely accept repaired associations as previously studied, we separately analyzed hits (i.e., correct endorsement of a repeated pair) and false alarms (i.e., incorrect endorsement of a rearranged pair) using repeated-measure ANOVAs with task run as a withinsubject factor and match group (low vs. high) as a betweensubject factor. For hits, none of the effects was statistically reliable, (all ps $>0.20$ ). For false alarms (Fig. 2B), there was a significant main effect of run, $F(2,56)=8.82, p=0.001, \eta_{p} 2=0.24$, but no main effect of group, $F(1,28)=1.54, p=0.22, \eta_{p} 2=0.05$. The run $\times$ match group interaction approached statistical significance, $F(2,56)=2.9$, $p=0.06, \eta_{p} 2=0.09$, suggesting a steeper decrease in the amount of false alarms in the high-match older adults.

These group differences were further corroborated by correlational analyses of individual deviation scores and task performance. Across the entire sample of older adults, higher match (i.e., more negative scores) was associated with higher overall recognition, $r=-0.53, p=0.003$ and lower false alarm rates, $r=0.36, p=0.05$. The correlation between deviation scores and hit rates was not reliable, $r=-0.11, p=0.57$.

In sum, high- and low-match older adults did not differ reliably in initial performance, but only high-match older adults enhanced their recognition performance in later runs of the task. Follow-up analyses revealed that these improvements reflected improved ability of high-match older adults to correctly detect repaired

Table 1

Descriptive characteristics and neuropsychological measures for high- and low-match groups

\begin{tabular}{|c|c|c|c|}
\hline & High-match older adults mean (SD) & Low-match older adults mean (SD) & Test of group differences \\
\hline Age & $71.75(1.83)$ & $72.81(2.09)$ & $t(28)=1.48, p=0.15$ \\
\hline Gender (females, males) & 7,8 & 6,9 & \\
\hline Digit symbol substitution test & $51.8(5.75)$ & $45.87(13.47)$ & $t(18.9)=1.57, p=0.13$ \\
\hline Spot-a-word vocabulary task & $30.00(1.77)$ & $27.73(3.73)$ & $t(28)=2.13, p=0.04$ \\
\hline Digit sorting task & $8.93(2.12)$ & $7.47(2.50)$ & $t(28)=1.73, p=0.10$ \\
\hline WCST (perseverative errors) & $16.97(7.71)$ & $22.68(7.87)$ & $t(28)=2.01, p=0.05$ \\
\hline
\end{tabular}

Key: SD, standard deviation; WCST, Wisconsin Card Sorting Task. 
A

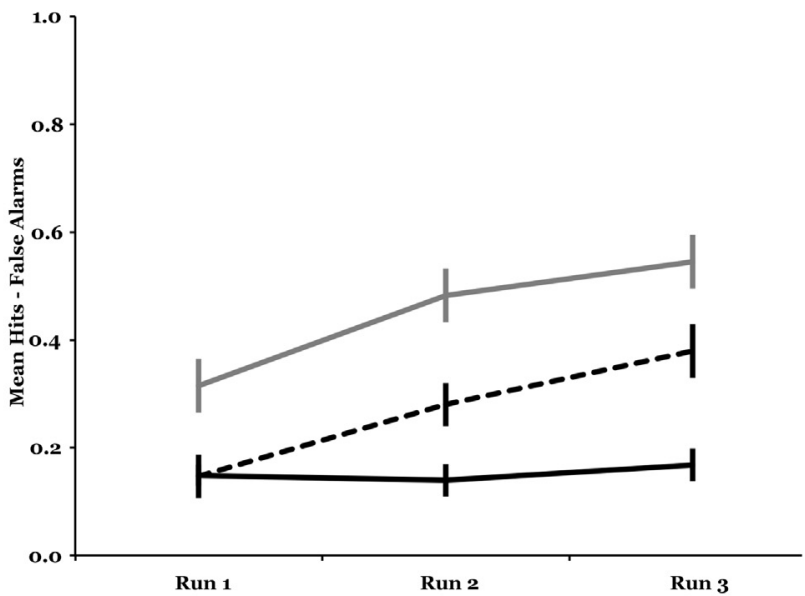

B

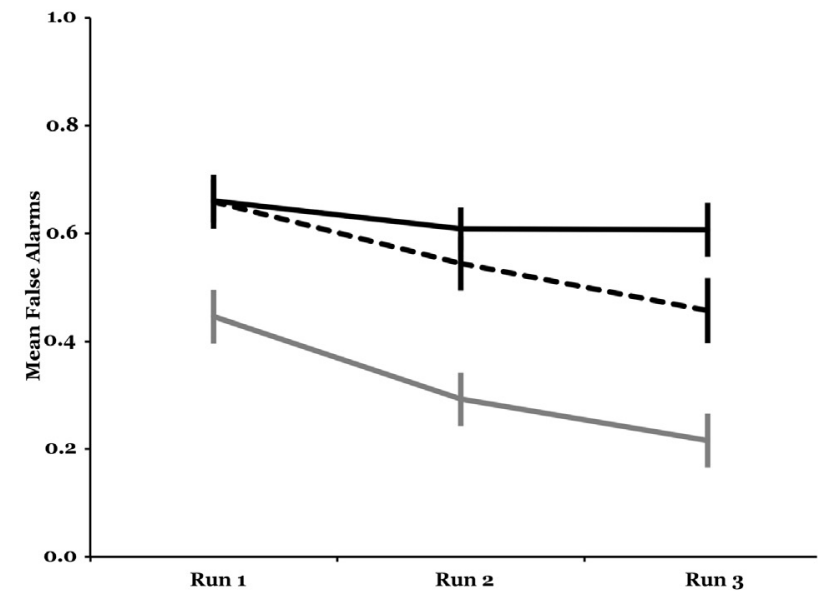

C

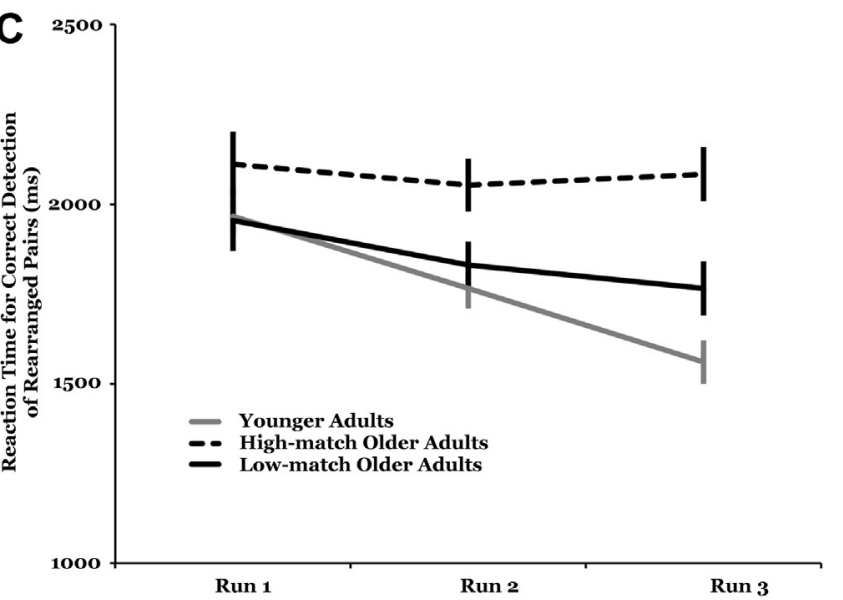

Fig. 2. Performance in the continuous recognition paradigm for high- and low-match older adults. (A) Overall recognition (hits-false alarms). (B) False alarm rates for rearranged pairs. Younger adults are plotted, but not included in the reported analyses. (C) Reaction times for correct detection of re-paired associations. Error bars represent standard error of the mean.

associations. This ability, which is particularly impaired in normal aging (Bender et al., 2010; Shing et al., 2008), appears to be recoverable in high-match older adults.

\subsection{Functional activation differences between high-match and low- match older adults}

The functional networks of high- and low-match older adults may differ in many ways. To address this issue, we directly compared the patterns of functional activation during correct rejection of repaired associations (i.e., CR rearranged $>$ CR novel) between high- and low-match older adults (Fig. 1). Compared with low-match older adults, high-match participants showed greater activation in bilateral superior parietal lobe, middle frontal, and right paracingulate gyrus (Table 2), that is, the regions of the average young-adult network. There were no regions in which lowmatch older adults demonstrated greater activation than highmatch older adults.

Direct comparisons between each match group and younger adults can be found in the Supplementary Table 1. In sum, compared with younger adults, there were no regions in which either low- or high-match groups showed higher activation. However, these results should be interpreted with some caution, given the smaller sample size of each of the older-adult match groups.

Taken together, compared with low-match older adults, highmatch older adults displayed stronger task-related modulation in a frontoparietal network when correctly detecting a repaired association. Low-match older adults did not show additional neural recruitment for detecting repaired associations. These results suggest that the differences between high- and low-match older adults resulted from underactivations in the low-match group. It is therefore possible that the relationship between memory performance and brain activation was actually driven by activation differences within task-related regions (i.e., inside the average younger adults' mask for rearranged pairs) or, alternatively, by activation differences within additional regions (i.e., outside of the average younger adults' mask), rather than by the difference score of these values. In fact, $T_{\text {inside }}$ mask was positively related to recognition sores in older adults, $r=0.34, p=0.07$, whereas the correlation between $T_{\text {outside mask }}$ and recognition scores was not reliable, $r=0.06, p=0.77$. To directly test whether these correlations were reliably different from the observed relationship between match (higher match represented by more negative deviation scores) and recognition performance $(r=-0.53, p=0.003$, see Section 3.2), we used the formulas described in Meng et al. (1992) (in the general case of the formula, correlations between correlated predictor variables and a common outcome can be tested using a $\chi^{2}$ test such that $\chi^{2}(\mathrm{k}-1)=\left((\mathrm{N}-3) \times \Sigma_{\mathrm{i}}\left(\mathrm{Z}_{\mathrm{ri}}-\mathrm{Z}_{\mathrm{r}}\right)^{2}\right) /\left(\left(1-\mathrm{r}_{\mathrm{x}}\right) \times \mathrm{h}\right)$, with $\mathrm{k}=$ number of tested correlations, $N=$ number of subjects, $Z_{\mathrm{ri}}=$ Fisher

Table 2

Neural activity for CR rearranged pairs $>$ CR novel pairs

\begin{tabular}{lrrrrrr}
\hline \multirow{2}{*}{ Region } & \multicolumn{7}{l}{ MNI coordinates $(\mathrm{mm})$} & & & \\
\cline { 2 - 7 } & $\mathrm{BA}$ & Voxels & $\mathrm{Z}$ max & $\mathrm{X}$ & $\mathrm{Y}$ & $\mathrm{Z}$ \\
\hline Younger adults & 7 & 9471 & 6.39 & -18 & -66 & 48 \\
$\quad$ L superior parietal lobe & 6 & 1492 & 5.6 & -30 & 8 & 54 \\
L middle frontal gyrus & 10 & 1361 & 4.75 & -46 & 54 & -4 \\
L anterior PFC & 8 & 825 & 4.65 & 4 & 24 & 48 \\
R superior frontal gyrus & 6 & 704 & 4.97 & 32 & 8 & 52 \\
R middle frontal gyrus & 23 & 265 & 4.67 & -2 & -26 & 28 \\
L posterior cingulate gyrus & $17 / 18$ & 79 & 3.51 & -6 & -70 & 8 \\
L intracalcarine cortex & & 75 & 4.04 & -8 & -16 & 16 \\
L thalamus & 13 & 27 & 3.46 & -32 & 24 & -2 \\
L insula & 17 & 24 & 3.32 & 14 & -90 & 4 \\
R occipital lobe & 17 & 13 & 3.2 & -12 & -84 & 10 \\
L occipital lobe & & 12 & 3.3 & 40 & -74 & 6 \\
R occipital lobe & & & & & \\
High-match > low-match older adults & & & & & \\
L superior parietal lobe & 7 & 4315 & 3.92 & -24 & -66 & 52 \\
L middle frontal gyrus & 9 & 4103 & 4.19 & -44 & 34 & 30 \\
R paracingulate gyrus & & 392 & 3.32 & 6 & 20 & 42 \\
R middle frontal gyrus & 9 & 279 & 3.28 & 48 & 32 & 30 \\
L insular cortex & & 195 & 2.95 & -28 & 24 & 0 \\
R middle frontal gyrus & & 77 & 2.84 & 26 & 12 & 60
\end{tabular}

Key: BA, Brodmann area; CR, correct rejections; L, left; MNI, Montreal Neurological Institute atlas; PFC, prefrontal cortex; R, right 
$Z$-transformed correlations, $Z_{r}=$ average $Z$ value of all tested correlations, $\mathrm{r}_{\mathrm{X}}=$ median intercorrelation among the predictor variables, $\mathrm{h}=\left(1-\mathrm{f} \times \mathrm{r}^{2}\right) /\left(1-\mathrm{r}^{2}\right)$, where $\mathrm{r}^{2}=$ mean of all tested correlation values, and $\left.\mathrm{f}=\left(1-\mathrm{r}_{\mathrm{x}}\right) / 2 \times\left(1-\mathrm{r}^{2}\right)\right)$. The correlations were significantly different from each other, $\chi^{2}(2)=8.46, p=0.01$. The correlation between recognition performance and deviation scores was reliably stronger than the correlation between recognition scores and $T_{\text {inside mask }}, t(27)=2.82, p=0.01$. Similarly, the relationship between recognition and deviation scores was stronger than the correlation between recognition scores and $T_{\text {outside }}$ mask, $t(27)=2.16, p=0.04$.

Taken together, these results suggest that greater average taskrelated activation within brain regions engaged by younger adults for correctly detecting repaired associations might be beneficial for older adults, whereas greater average task-related activation outside the younger-adult mask do not carry any benefit by itself. Importantly, the difference in task-related activation between inside and outside the younger-adult mask, as captured by the deviation score, was more strongly associated with successful associative recognition in later adulthood than any of the component scores.

\subsection{Functional connectivity differences between high-match and low-match older adults}

To further delineate the nature of individual differences in correctly detecting repaired associations among the older adults of the present study, we examined the association of functional activation match to younger adults with functional connectivity of the left anterior PFC, a region that plays a central role in mnemonic control of highly familiar information (Fandakova et al., 2014). The results of the PPI analysis revealed that the left anterior PFC seed region was more highly connected to the right postcentral, parahippocampal, and middle temporal gyrus in the high-match group than in the low-match group during correct rejection of rearranged pairs (see Table 3). There were no regions that displayed stronger functional connectivity in low-match older adults. To verify that the results from the PPI analysis were not confounded by differences in motion, we calculated framewise displacement following the procedure described by Power et al. (2012). Mean framewise displacement across all volumes did not differ between low-match $(\mathrm{M}=0.82, \mathrm{SE}=0.10)$ and high-match older adults $(\mathrm{M}=0.78, \mathrm{SE}=$ $0.11), t(28)=0.21, p=0.83$.

To better understand these connectivity differences, we examined the connectivity pattern of younger adults. During correct

Table 3

Functional connectivity with left anterior PFC for CR rearranged pairs $>$ CR novel pairs

\begin{tabular}{lccrrrr}
\hline Region & \multicolumn{7}{l}{ MNI coordinates $(\mathrm{mm})$} & & & \\
\cline { 2 - 7 } & $\mathrm{BA}$ & Voxels & $\mathrm{Z}$ max & $\mathrm{X}$ & $\mathrm{Y}$ & $\mathrm{Z}$ \\
\hline High-match > low-match older adults & & & & & \\
R postcentral gyrus & & 548 & 4.19 & 38 & -26 & 70 \\
R middle temporal gyrus & & 104 & 2.77 & 70 & -26 & -14 \\
L occipital fusiform gyrus & & 49 & 3.20 & -32 & -70 & -18 \\
R parahippocampal gyrus & & 30 & 2.75 & 36 & -24 & -22 \\
L precentral gyrus & & 29 & 2.62 & -34 & -14 & 46 \\
Younger adults & & & & & & \\
R inferior parietal lobe & 19 & 135 & 3 & 26 & -80 & 56 \\
L inferior temporal lobe & & 61 & 3.26 & -62 & -62 & -12 \\
L inferior parietal lobe & & 53 & 2.99 & -16 & -62 & 28 \\
R frontal pole & 10 & 53 & 2.85 & 38 & 58 & -18 \\
L middle frontal gyrus & $44 / 45$ & 51 & 2.61 & -54 & 20 & 36 \\
L middle frontal gyrus & 9 & 43 & 2.98 & -40 & 28 & 30 \\
L inferior parietal lobe & & 40 & 3.06 & -64 & -50 & 44 \\
\hline
\end{tabular}

Key: BA, Brodmann area; CR, correct rejections; L, left; MNI, Montreal Neurological Institute atlas; PFC, prefrontal cortex; R, right. detection of repaired associations, younger adults demonstrated stronger connectivity between left anterior PFC and frontal gyrus, inferior parietal, and temporal areas (see Table 3). A direct comparison of the match groups and younger adults can be found in Supplementary Table 2. Taken together, the brain areas in which high-match older adults showed stronger connectivity than lowmatch older adults were not part of the functional connectivity network observed in younger adults. This suggests that high-match older adults might have adopted a different strategy than younger adults to detect repaired associations, such as relying more on semantic elaboration and/or exact reinstatement of the original associations supported by connectivity between anterior PFC and posterior brain regions.

These results indicate that differences in the degree to which functional activation among older adults matched the networks engaged by younger adults was related to differences in functional connectivity, over, and above main task effects. One possible reason for these differences may be more controlled and detailed processing of the repaired associations in the high-match older adults. This idea is supported when examining the reaction times for these decisions. A repeated-measures ANOVA on the reaction times for correctly rejected repaired associations with run as a withinsubject factor and group (high-match older adults, low-match older adults, and younger adults) as a between-subject factor revealed a reliable effect of run, $F(2,110)=20.27, p<0.001, \eta_{p} 2=$ 0.27 , group, $F(2,55)=7.05, p=0.002, \eta_{p} 2=0.20$, as well as a group $\times$ run interaction, $F(4,110)=6.51, p<0.001, \eta_{p} 2=0.19$. Post hoc comparisons indicated no differences among the groups in the first run, $F(2,55)=1.32, p=0.28, \eta_{p} 2=0.05$, when rejecting repaired associations was expected to be most challenging. In the second run the difference among the groups, $F(2,55)=4.97, p=$ $0.01, \eta_{p} 2=0.15$, was driven by longer response times in high-match older adults compared with younger adults, $t(41)=3.02, p=0.008$, $d=0.97$. In the last run the groups differed, $F(2,55)=14.32, p<$ $0.001, \eta_{p} 2=0.34$, because of longer reaction times in high-match older adults compared to both younger adults, $t(41)=5.3, p<$ $0.001, d=1.71$, and to low-match older adults, $t(28)=2.99, p=0.02$, $d=1.09$ (Fig. 2C). Importantly, there were no reaction time differences among the groups for any other condition, including hits and correct rejections of novel word pairs (all ps > 0.05).

\subsection{Free recall differences between high-match and low-match older adults}

To further characterize the difference between high- and lowmatch older adults in strategic memory, we examined their performance differences using the California Verbal Learning Task (CVLT; Delis et al., 1987). In this standardized measure of free recall, 16 words from 4 semantic categories are presented in mixed order for a total of 5 repetitions, each of them followed by a free recall test. Findings from patients with PFC lesions suggest that this task taps on strategic memory use that depends on PFC functioning (Alexander et al., 2003; Baldo et al., 2002). Importantly, selfinitiated use of memory strategies can be explicitly assessed in the CVLT by a clustering measure that captures the tendency to recall items from the same category in consecutive order (Roenker et al., 1971). We expected that if our match scores capture individual differences in strategic aspects of memory, which engage frontoparietal networks, then these scores should predict differences in both performance and strategic behavior on an independently assessed task that puts high demands on mnemonic control (cf. de Chastelaine et al., 2011).

Across the 5 CVLT repetitions, high-match older adults $(M=14$, $\mathrm{SE}=0.27)$ recalled more words than low-match older adults $(\mathrm{M}=$ 12.9, $\mathrm{SE}=0.25), F(1,28)=6.37, p=0.02, \eta_{p} 2=0.19$. Mean 
differences in recall were accompanied by differences in strategic clustering such that high-match older adults $(\mathrm{M}=0.50, \mathrm{SE}=0.06)$ were almost twice more likely to use categorical clustering at retrieval than low-match older adults $(\mathrm{M}=0.27, \mathrm{SE}=0.07)$, $F(1,28)=6.63, p=0.02, \eta_{p} 2=0.19$. Categorical clustering accounted for variance in free recall beyond the effect of match group, $R_{\text {change }}^{2}=0.18, F_{\text {change }}(1 / 27)=7.9, p=0.01$, but showed no interaction with match group $(p>0.05)$, indicating that categorical clustering is an important predictor of CVLT performance in both high- and low-match individuals.

\section{Discussion}

This study yielded 3 main findings. First, among older adults, higher match to the average younger-adult frontoparietal network engaged in the detection of repaired associations was related to better associative recognition (particularly, less false alarms), more pronounced improvement across runs of a continuous recognition task that placed relatively high demands on mnemonic control as well as superior strategic recall in an independent memory task. Second, older adults with lower match showed attenuated taskrelated signals in the frontoparietal mnemonic network, and no evidence for additional recruitment of regions outside this network. Third, high- and low-match older adults showed different patterns of functional connectivity during detection of repaired associations, with high-match individuals demonstrating stronger connectivity between anterior PFC and parahippocampal as well as middle temporal gyrus.

Among older adults, more youth-like activation during the correct recognition of repaired associations was associated with less false endorsements of repaired associations. In contrast, no behavioral differences were found in the correct detection of exact repeats of studied word pairs. These findings are in line with previous reports that aging affects false alarms in associative recognition paradigms to a greater degree than hits (e.g., Bender et al., 2010; Shing et al., 2008), indicating a specific age-related deficit in rejecting novel configurations of familiar information. Highmatch older adults also showed a more pronounced benefit from the repetition of original word pairs across runs and correctly rejected more novel configurations of these word pairs in later runs. Recently, Kilb and Naveh-Benjamin (2011) reported that pair (but not single item) repetitions led to gains in associative memory among older adults that are comparable with the benefit observed in younger adults. These gains were associated with a reduced tendency to falsely endorse rearranged configurations. Here, we find that greater similarity of older adults' mnemonic control network to the young-adult pattern is associated with preserved potential for memory improvement and a more flexible use of mnemonic control mechanisms, resulting in better adaptation to changing environmental demands (cf. de Chastelaine et al., 2011; Fandakova et al., 2012; Wang et al., 2009). Based on these sets of results, it seems likely that the repetition of pairs in the Kilb and Naveh-Benjamin (2011) study functioned as a form of environmental support (Craik, 1983; Lindenberger and Mayr, 2014) that reduced the demands on mnemonic control mechanisms in both age groups.

Our results also demonstrate that the observed variability in behavioral responses across individuals was related to differences in neural activation. The relevant activations were confined to a frontoparietal network engaged in the recollection of episodic information (e.g., Spaniol et al., 2009) and the rejection of repaired associations in particular (e.g., Lepage et al., 2003). This frontoparietal brain network bears close resemblance with the processgeneral cognitive network that was recently identified across encoding and retrieval of face-name pairs in a large sample of 25- to 80-year-olds (Salami et al., 2012). In this study, older adults who activated the process-specific encoding network less engaged the process-general cognitive network more. Likewise, the high-match older adults in the present study may possess more neural resources to modulate the process-general cognitive network in response to changing environmental demands (e.g., Gazzaley et al., 2008).

We found no evidence for additional neural recruitment in the low- or high-match older adults compared with younger adults. Thus, our findings do not provide evidence in favor of compensatory activation as a marker of successful aging but are more in line with evidence that preserved functional activation in older adults is associated with higher memory performance (Düzel et al., 2011; Nagel et al., 2009, 2011). Although the present results are based on cross-sectional evidence, a recent longitudinal study found considerable variability in episodic memory across 20 years in 55to 79-year-olds, such that some declined whereas others remained stable (Persson et al., 2012). Of particular interest, only individuals with declining performance demonstrated declines in mediotemporal activation across a 6-year period, suggesting that preserved memory ability was associated with preserved functional activation of regions within the episodic memory network. Other studies have found that functional maintenance and preserved cognitive functions are tightly coupled to structural maintenance (Burzynska et al., 2013). Taken together, the present results provide support for the recently introduced concept of brain maintenance in later adulthood (Lindenberger et al., 2013; Nyberg et al., 2012), according to which individuals who better maintain task-related brain characteristics are the ones that demonstrate less memory decline.

In this context, the results of the present PPI analysis suggest that individual differences in the degree of maintenance of functional activation in later adulthood map onto differences in functional connectivity. High-match older adults showed stronger task-related connectivity between left anterior PFC and middle temporal and parahippocampal gyrus than low-match older adults. During false memory retrieval, Dennis et al. (2008b) found increased activity in older relative to younger adults in middle temporal gyrus and anterior PFC, presumably reflecting internal generation of associations between semantically related stimuli. The parahippocampal gyrus plays a central role in the encoding and retrieval of associative, and especially contextual, information (Aminoff et al., 2013; Diana et al., 2007). Taken together, the stronger functional connectivity of left anterior PFC with the parahippocampal and the middle temporal gyrus in high-match older adults may reflect differences in mnemonic control processes during detection of repaired associations, in particular the search for or further elaboration of stored contextual representations. In line with this interpretation, highmatch older adults took longer than low-match older adults to come to a decision when correctly rejecting repaired associations, likely reflecting a more controlled evaluation of the stimuli. These results add to previous studies demonstrating adult age differences in functional connectivity (Daselaar et al., 2006; Nagel et al., 2011; St. Jacques et al., 2009) and point to the sizeable heterogeneity of neural correlates of memory functioning in later adulthood (Lindenberger et al., 2013).

To further validate our findings, we tested whether higher match to younger adults' functional activation networks for detecting repaired associations was related to performance on an independently assessed memory task that presumably requires similar mnemonic control process, the CVLT. Computational models of the CVLT suggest that lesions to the PFC module would lead to lower levels of recall and less categorical clustering (Becker and Lim, 2003). This corresponds precisely to what we observed in low-match older adults, relative to high-match older adults. Our 
findings are consistent with recent study by Kirchhoff et al. (2014), who found that age differences in semantic clustering are associated with PFC gray matter volume. Another study with 20- to 80year-olds showed that semantic clustering is associated with functional activations during encoding and subsequent free recall (Hazlett et al., 2010). These findings support our expectation that individual differences in mnemonic control are an important source of heterogeneity in associative memory among older adults. Future research needs to examine the extent to which the observed heterogeneity reflects process-general or process-specific differences. A closer examination of the administered cognitive tests (Table 1) suggests that high-match older adults showed superior performance not only on tasks related to executive functioning but also on vocabulary knowledge, raising the possibility that the observed individual differences may reflect a domain-general mechanism (cf. Fandakova et al., 2012). Alternatively, given that we used verbal materials that rely on semantic processing, the match group differences in vocabulary may reflect superior preserved semantic processing in high-match older adults.

The present findings demonstrate the validity of deviation scores for characterizing individual differences in functional activation during the correct detection of repaired associations among older adults. In doing so, they lend further credence to earlier studies that have advocated the use of deviation scores to capture individual differences in brain structure (Seeley et al., 2009) and brain function (Düzel et al., 2011) during episodic encoding. Future studies should explore the validity and generalizability of deviation scores for other paradigms, cognitive domains, and group comparisons.

It is worth noting that the deviation score used in the present study is a global measure of similarity and deviation. The average young-adult network engaged in the correct rejection of repaired associations comprised a number of different anatomic regions including PFC, parietal, and occipital regions that support different cognitive operations and may differ in their relative contribution to the detection of repaired associations. These relative differences are partly captured in the deviation score as regions with stronger taskrelated activation will contribute more to the mean $T$-value relative to regions with weaker task-related activation. However, the deviation score does not capture the degree to which the relative contributions of individual anatomic regions differ across older adults. For example, it is possible that using different strategies to detect repaired associations may affect the relative weighting of the regions within the functional network, as suggested by the results of the connectivity analysis. An important task for future studies is to determine the extent to which the relative task-specific hierarchy of different anatomic regions is maintained in later adulthood. The present results suggest that one important aspect contributing to individual differences in associative memory may be differences in task-related functional connectivity, even when activation levels are similar.

The results of the present study indicate that individual differences in strategic memory processing in a frontoparietal network contribute to individual differences in associative memory in healthy cognitive aging. The present results extend our previous report of mean differences between groups of healthy younger and older adults (Fandakova et al., 2014) by identifying neural mechanisms of individual differences in memory performance within the group of older adults. Evidence from functional activation, connectivity, and behavior converge on the notion that the maintenance of youth-like brain functions may promote successful detection of novel configurations of familiar information, (cf. Lindenberger et al., 2013; Nyberg et al., 2012). Longitudinal evidence is needed to further corroborate the notion that maintenance is an important general factor in promoting high-level cognitive functioning in later adulthood.

\section{Disclosure statement}

The authors have no conflicts of interest to disclose.

\section{Appendix A. Supplementary data}

Supplementary data associated with this article can be found, in the online version, at http://dx.doi.org/10.1016/j.neurobiolaging. 2014.10.022.

\section{References}

Alexander, M.P., Stuss, D.T., Fansabedian, N., 2003. California Verbal Learning Test: performance by patients with focal frontal and non-frontal lesions. Brain 126 1493-1503.

Aminoff, E.M., Kveraga, K., Bar, M., 2013. The role of the parahippocampal cortex in cognition. Trends Cogn. Sci. 17, 379-390.

Badre, D., Wagner, A.D., 2005. Frontal lobe mechanisms that resolve proactive interference. Cereb. Cortex 15, 2003-2012.

Baldo, J.V., Delis, D., Kramer, J., Shimamura, A.P., 2002. Memory performance on the California Verbal Learning Test-II: findings from patients with focal frontal lesions. J. Int. Neuropsychol. Soc. 8, 539-546.

Barulli, D.J., Stern, Y., 2013. Efficiency, capacity, compensation, maintenance: emerging concepts in cognitive reserve. Trends Cogn. Sci. 17, 502-509.

Becker, S., Lim, J., 2003. A computational model of prefrontal control in free recall: strategic memory use in the California Verbal Learning Task. J. Cogn. Neurosci. 15, 821-832.

Bender, A.R., Naveh-Benjamin, M., Raz, N., 2010. Associative deficit in recognition memory in a lifespan sample of healthy adults. Psychol. Aging 25, 940-948.

Burzynska, A.Z., Garrett, D.D., Preuschhof, C., Nagel, I.E., Li, S.C., Bäckmann, L., Heekeren, H.R., Lindenberger, U., 2013. A scaffold for efficiency in the human brain. J. Neurosci. 33, 17150-17159.

Cabeza, R., Dennis, N.A., 2013. Frontal lobes and aging: deterioration and compensation. In: Stuss, D.T., Knight, R.T. (Eds.), Principles of Frontal Lobe Function, second ed. Oxford University Press, New York, pp. 628-652.

Craik, F.I.M., 1983. On the transfer of information from temporary to permanent memory, Philos. Trans. R. Soc. Lond. B: Biol. Sci. 302, 341-359.

Dale, A.M., 1999. Optimal experimental design for event-related fMRI. Hum. Brain Mapp. 8, 109-114.

Daselaar, S.M., Fleck, M.S., Dobbins, I.G., Madden, D.J., Cabeza, R., 2006. Effects of healthy aging on hippocampal and rhinal memory functions: an event-related fMRI study. Cereb. Cortex 16, 1771-1782.

de Frias, C.M., Lovden, M., Lindenberger, U., Nilsson, L.G., 2007. Revisiting the dedifferentiation hypothesis with longitudinal multi-cohort data. Intelligence 35, 381-392.

de Chastelaine, M., Wang, T.H., Minton, B., Muftuler, L.T., Rugg, M.D., 2011. The effects of age, memory performance, and callosal integrity on the neural correlates of successful associative encoding. Cereb. Cortex 21, 2166-2176.

Delis, D.C., Kramer, J.H., Kaplan, E., Ober, B.A., 1987. The California Verbal Learning Test: Research Edition. Psychological Corporation, New York.

Dennis, N.A., Hayes, S.M., Prince, S.E., Madden, D.J., Huettel, S.A., Cabeza, R., 2008 a. Effects of aging on the neural correlates of successful item and source memory encoding. J. Exp. Psychol. Learn. Mem. Cogn. 34, 791-808.

Dennis, N.A., Kim, H., Cabeza, R., 2008b. Age-related differences in brain activity during true and false memory retrieval. J. Cogn. Neurosci. 20, 1390-1402.

Diana, R.A., Yonelinas, A.P., Ranganath, C., 2007. Imaging recollection and familiarity in the medial temporal lobe: a three-component model. Trends Cogn. Sci. 11, $379-386$.

Düzel, E., Schütze, H., Yonelinas, A.P., Heinze, H.J., 2011. Functional phenotyping of successful aging in long-term memory: preserved performance in the absence of neural compensation. Hippocampus 21, 803-814.

Fandakova, Y., Lindenberger, U., Shing, Y.L., 2014. Deficits in process-specific prefrontal and hippocampal activations contribute to adult age differences in episodic memory interference. Cereb. Cortex 24, 1832-1844.

Fandakova, Y., Shing, Y.L., Lindenberger, U., 2012. Heterogeneity in memory training improvement among older adults: a latent class analysis. Memory 20, 554-567.

Ford, J.H., Verfaellie, M., Giovanello, K.S., 2010. Neural correlates of familiarity-based associative retrieval. Neuropsychologia 48, 3019-3025.

Friston, K.J., Buechel, C., Fink, G.R., Morris, J., Rolls, E., Dolan, R.J., 1997. Psychophysiological and modulatory interactions in neuroimaging. Neuroimage 6, 218-229.

Gazzaley, A., Clapp, W., McEvoy, K., Knight, R., D’Esposito, M., 2008. Age-related topdown suppression deficit in the early stages of cortical visual memory processing. Proc. Natl. Acad. Sci. U. S. A. 105, 13122-13126.

Ghisletta, P., Rabbitt, P., Lunn, M., Lindenberger, U., 2012. Two thirds of the agebased changes in fluid and crystallized intelligence, perceptual speed, and memory in adulthood are shared. Intelligence 40, 260-268.

Giovanello, K.S., Schacter, D.L., 2012. Reduced specificity of hippocampal and posterior ventrolateral prefrontal activity during relational retrieval in normal aging. J. Cogn. Neurosci. 24, 159-170. 
Hazlett, E.A., Byne, W., Brickman, A.M., Mitsis, E.M., Newmark, R., Haznedar, M.M., Knatz, D.T., Chen, A.D., Buchsbaum, M.S., 2010. Effects of sex and normal aging on regional brain activation during verbal memory performance. Neurobiol. Aging 31, 826-838.

Jacoby, L.L., Rhodes, M.G., 2006. False remembering in the aged. Curr. Dir. Psychol. Sci. 15, 49-53.

Kilb, A., Naveh-Benjamin, M., 2011. The effects of pure pair repetition on younger and older adults' associative memory. J. Exp. Psychol. Learn. Mem. Cogn. 37, 706-719.

Kirchhoff, B.A., Gordon, B.A., Head, D., 2014. Prefrontal gray matter volume mediates age effects on memory strategies. Neuroimage 90, 326-334.

Lepage, M., Brodeur, M., Bourgouin, P., 2003. Prefrontal cortex contribution to associative recognition memory in humans: an event-related functional magnetic resonance imaging study. Neurosci. Lett. 346, 73-76.

Lindenberger, U., Burzynska, A.Z., Nagel, I.E., 2013. Heterogeneity in frontal lobe aging. In: Stuss, D.T., Knight, R.T. (Eds.), Principles of Frontal Lobe Function, second ed. Oxford University Press, New York, pp. 609-627.

Lindenberger, U., Ghisletta, P., 2009. Cognitive and sensory declines in old age: gauging the evidence for a common cause. Psychol. Aging 24, 1-16.

Lindenberger, U., Mayr, U., 2014. Cognitive aging: Is there a dark side to environmental support? Trends Cogn Sci 18, 7-15.

Meng, X.L., Rosenthal, R., Rubin, D.B., 1992. Comparing correlated correlation coefficients. Psych. Bull. 111, 172-175.

Mitchell, K.J., Johnson, M.K., 2009. Source monitoring 15 years later: what have we learned from fMRI about the neural mechanisms of source memory? Psychol. Bull. 135, 638-677.

Nagel, I.E., Preuschhof, C., Li, S.C., Nyberg, L., Backman, L., Lindenberger, U., Heekeren, H.R., 2011. Load modulation of BOLD response and connectivity predicts working memory performance in younger and older adults. J. Cogn. Neurosci. 23, 2030-2045.

Nagel, I.E., Preuschhof, C., Li, S.C., Nyberg, L., Backman, L., Lindenberger, U., Heekeren, H.R., 2009. Performance level modulates adult age differences in brain activation during spatial working memory. Proc. Natl. Acad. Sci. U. S. A. 106, 22552-22557.

Naveh-Benjamin, M., 2000. Adult age differences in memory performance: tests of an associative deficit hypothesis. J. Exp. Psychol. Learn. Mem. Cogn. 26, 1170-1187.

Nyberg, L., Lövden, M., Riklund, K., Lindenberger, U., Bäckman, L., 2012. Memory aging and brain maintenance. Trends Cogn. Sci. 16, 292-305.

Old, S.R., Naveh-Benjamin, M., 2008. Differential effects of age on item and associative measures of memory: a meta-analysis. Psychol. Aging 23, 104-118.
Persson, J., Pudas, S., Lind, J., Kauppi, K., Nilsson, L.G., Nyberg, L., 2012. Longitudinal structure-function correlates in elderly reveal MTL dysfunction with cognitive decline. Cereb. Cortex 22, 2297-2304.

Power, J.D., Barnes, K.A., Snyder, A.Z., Schlaggar, B.L., Petersen, S.E., 2012. Spurious but systematic correlations in functional connectivity MRI networks arise from subject motion. Neuroimage 59, 2142-2154.

Raz, N., Lindenberger, U., Rodrigue, K.M., Kennedy, K.M., Head, D., Williamson, A Dahle, C., Gerstorf, D., Acker, J.D., 2005. Regional brain changes in ageing healthy adults: general trends, individual differences and modifiers. Cereb. Cortex 15 1676-1689.

Reuter-Lorenz, P.A., Cappell, K.A., 2008. Neurocognitive aging and the compensation hypothesis. Curr. Dir. Psychol. Sci. 17, 177-182.

Roenker, D.L., Brown, S.C., Thompson, C.P., 1971. Comparison of measures for estimation of clustering in free recall. Psych. Bull. 76, 45-48.

Salami, A., Eriksson, J., Nyberg, L., 2012. Opposing effects of aging on large-scale brain systems for memory encoding and cognitive control. J. Neurosci. 23 10749-10757.

Seeley, W.W., Crawford, R.K., Zhou, J., Miller, B.L., Greicius, M.D., 2009. Neurodegenerative diseases target large-scale human brain networks. Neuron 62 $42-52$.

Shing, Y.L., Werkle-Bergner, M., Brehmer, Y., Muller, V., Li, S.C., Lindenberger, U. 2010. Episodic memory across the lifespan: the contributions of associative and strategic components. Neurosci. Biobehav. Rev. 34, 1080-1091.

Shing, Y.L., Werkle-Bergner, M., Li, S.C., Lindenberger, U., 2008. Associative and strategic components of episodic memory: a life-span dissociation. J. Exp. Psychol. Gen. 137, 495-513.

Simons, J.S., Spiers, H.J., 2003. Prefrontal and medial temporal lobe interactions in long-term memory. Nat. Rev. Neurosci. 4, 637-648.

Spaniol, J., Davidson, P.S.R., Kim, A.S.N., Han, H., Moscovitch, M., Grady, C.L. 2009. Event-related fMRI studies of episodic encoding and retrieval: metaanalyses using activation likelihood estimation. Neuropsychologia 47, 1765-1779.

Spencer, W.D., Raz, N., 1995. Differential effects of ageing on memory for conten and context: a meta-analysis. Psychol. Aging 10, 527-539.

St. Jacques, P.L., Dolcos, F., Cabeza, R., 2009. Effects of aging on functional connectivity of the amygdala for subsequent memory of negative pictures: A network analysis of fMRI data. Psycholo. Sci. 20, 74-84.

Wang, T.H., Kruggel, F., Rugg, M.D., 2009. Effects of advanced aging on the neural correlates of recognition memory. Neuropsychologia 47, 1352-1361. 